Magnetohydrodynamic convection in molten gallium

Juel, A. and Mullin, T. and Ben Hadid, H. and Henry, D.

1999

MIMS EPrint: 2007.132

Manchester Institute for Mathematical Sciences

School of Mathematics

The University of Manchester

\footnotetext{
Reports available from: http://eprints.maths.manchester.ac.uk/

And by contacting: The MIMS Secretary

School of Mathematics

The University of Manchester

Manchester, M13 9PL, UK
} 


\title{
Magnetohydrodynamic convection in molten gallium
}

\author{
By A. JUEL ${ }^{1} \dagger$, T. MULLIN ${ }^{1}$, H. BEN HADID ${ }^{2}$ \\ AND D. HENR Y ${ }^{2}$ \\ ${ }^{1}$ Schuster Laboratory, The University of Manchester, Manchester M13 9PL, UK \\ ${ }^{2}$ Laboratoire de Mécanique des Fluides et d'Acoustique UMR 5509, Ecole Centrale de \\ Lyon / Université Claude Bernard Lyon 1, BP 163, 69131 Ecully Cedex, France
}

(Received 6 February 1998 and in revised form 24 July 1998)

We present the results of an experimental and numerical study of the effects of a steady magnetic field on sidewall convection in molten gallium. The magnetic field is applied in a direction which is orthogonal to the main flow which reduces the convection and good agreement is found for the scaling of this effect with the relevant parameters. Moreover, qualitatively similar changes in the structure of the bulk of the flow are observed in the experiment and the numerical simulations. In particular, the flow is restricted to two dimensions by the magnetic field, but it remains different to that found in two-dimensional free convection calculations. We also show that oscillations found at even greater temperature gradients can be suppressed by the magnetic field.

\section{Introduction}

In crystal growth processes the temperature gradient between the melt and the solid gives rise to buoyancy-driven convection. In most practical situations the motion so induced is found to be highly disordered or even turbulent. It is known that these flows can produce irregular distributions of dopant called striations in the crystallized host material and this inhomogeneity is undesirable if one wants to grow good quality semiconductor crystals. Hence there is considerable interest in methods of suppressing or controlling fluid convection.

One method of controlling the convection is to apply an external magnetic field as reviewed by Series \& Hurle (1991). This induces an electromotive field which can be non-uniform in regions in the melt. Hence electrical currents can flow, and these interact with the applied magnetic field to damp the convective motion. These effects can be calculated explicitly for simple geometries but laboratory and practical flows are extremely complicated and require numerical computations. It is the aim of the present study to investigate the fundamental magnetohydrodynamic interactions in a simplified crystal growth geometry using a combined experimental and numerical approach. By doing this we hope to gain insight into the basic fluid mechanical processes and thereby provide a platform on which to build an understanding of more practical flows.

The laboratory model we have chosen to investigate is based on a horizontal Bridgman crystal growth geometry. In this configuration, a crucible containing the

$\dagger$ Present address: Center for Nonlinear Dynamics and Department of Physics, University of Texas at Austin, Austin, TX 78712, USA. 


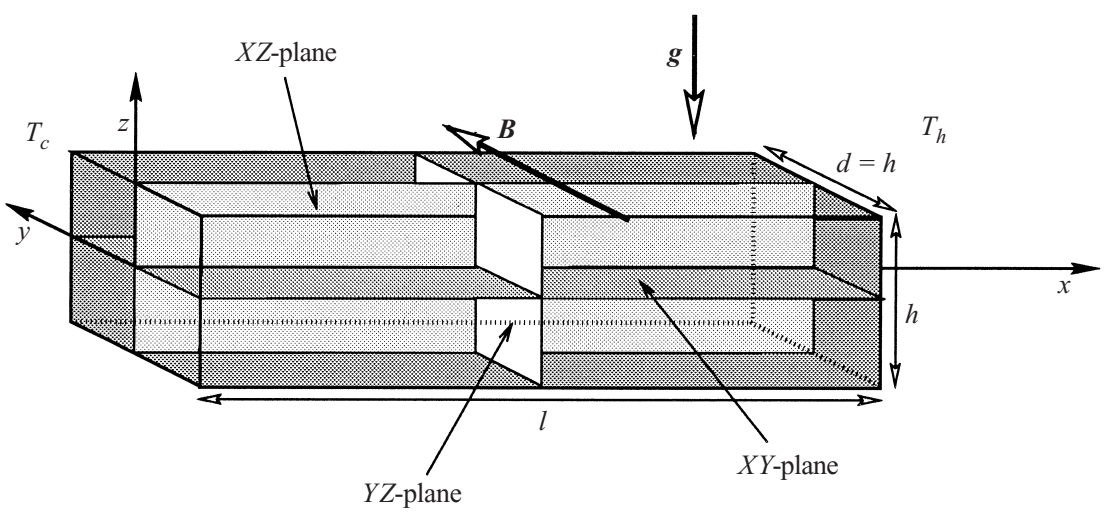

FIGURE 1. A schematic diagram of the geometry and coordinate system.

melt to be crystallized is slowly withdrawn from a hot oven so that a lengthwise temperature gradient arises and this drives convection in the liquid phase. This technique is not as commonly used as the Czochralski method where a seed crystal is pulled vertically from the melt contained in a heated crucible (Langlois 1985) but it is a technique which is of importance in growing crystals of specific form for instance (Müller \& Ostrogorsky 1993). Our laboratory system comprised a rectangular insulated boat of square cross-section which contained liquid gallium. It was heated and cooled in a controlled way at two opposite ends and a steady transverse magnetic field was applied using Helmholtz coils. A schematic diagram of the geometry is shown in figure 1. Complementary numerical investigations were performed using the spectral element techniques developed by Ben Hadid \& Henry (1997).

The pioneering experimental work on this problem was carried out by Hurle (1966) and Hurle, Jakeman \& Johnson (1974). They observe thermal oscillations in the flow and show that a magnetic field applied orthogonal to the main convective flow can be used to damp the time-dependence. However, a more recent investigation by McKell et al. (1990) reports that an applied magnetic field may also promote instabilities including low-dimensional chaos. Hence there is clearly a need for an in-depth study of the interaction between the flowing conductor and the applied magnetic field. We have chosen to investigate steady flows first since even here there are some interesting fluid mechanical questions to be addressed.

The steady flow in the absence of a magnetic field has been the subject of extensive theoretical and numerical investigations as reported in Daniels, Blythe \& Simpkins (1987) and Roux (1990). Particular attention is drawn to the article by Afrid \& Zebib (1990) since they emphasized the importance of three-dimensional effects in this flow. More recently, a detailed experimental and numerical investigation was carried out by Braunsfurth et al. (1997). They find good quantitative agreement between calculation and experiment and show that the principal cellular circulation has a two-dimensional form at modest temperature differences. However, more recent calculations and experiments by Juel et al. (1998) have revealed that there are three-dimensional effects ever-present in the flow field of the experiment and a full understanding of these may be required to make progress with the magnetohydrodynamic problem.

The two main parameters which govern the MHD flow are the Grashof number, $G r$, and Hartmann number, $\mathrm{Ha}$. Gr gives a measure of the relative importance of buoyancy to viscous forces and corresponds to the non-dimensional temperature gradient. $\mathrm{Ha}$ is a ratio between the Lorentz and viscous forces and is proportional to the strength 
of the imposed magnetic field. A third relevant quantity is the Prandtl number, $P r$, which is the ratio of viscous to thermal diffusivities. It is approximately zero for gallium which is a liquid metal above $29.8^{\circ} \mathrm{C}$ and hence heat transport principally takes place by conduction. However, as pointed out by Braunsfurth \& Mullin (1996) the qualitative form of observed oscillatory motion can depend sensitively on its precise value. Thus our calculations will be carried out at realistic finite values of $\mathrm{Pr}$, rather than the mathematically convenient but singular limit of zero.

There is an extensive literature on theoretical investigations of the damping of fluid motion by a magnetic field as reviewed by Hunt \& Shercliff (1971) and Moreau (1990). At large $H a$, inertia can be neglected and the flow field can be split into a core region where shear stresses are negligible compared with Lorentz forces and thin boundary layers. Alboussière, Garandet \& Moreau (1996) derive expressions for both the velocity and the induced current density in the presence of a transverse magnetic field, and predict that in our geometry, these should vary as $\mathrm{Ha}^{-1}$ and $\mathrm{Ha}^{-2}$ respectively.

So called 'Hartmann layers' which have thickness $O\left(\mathrm{Ha}^{-1}\right)$ are formed along the boundaries which are orthogonal to the direction of the applied magnetic field, i.e. along the two main side faces in our configuration. These interact with the core flow and provide a path for the electric currents to circulate within the cavity, as confirmed in the three-dimensional calculations by Ben Hadid \& Henry (1994). 'Parallel layers' which have thickness $O\left(\mathrm{Ha}^{-1 / 2}\right)$ are formed on those boundaries which lie parallel to the applied field. These are the top and bottom surfaces and the endwalls in our geometry. The parallel layers play a passive role in the problem since they are not directly affected by the magnetic field but they may contain significant velocity components.

Much of the modelling work on magnetohydrodynamic convection has been concerned with alternative configurations to the above one. The primary interest is in vertically and longitudinally oriented magnetic fields, i.e. parallel to gravity and to the applied temperature gradient respectively. A substantial advantage of these configurations is that they may be investigated using a two-dimensional analysis such as that performed by Singh \& Cowling (1963). A more modern example of this is given by Oreper \& Szekely (1983) who report the results of numerical calculations of sidewall convection in a square cavity when a longitudinal uniform magnetic field is imposed. They find that the magnetic field reduces the convective flow, but a large field does not suppress it completely. Instead a high field breaks the coupling between momentum and heat transport, which modifies the structure of the flow.

The effects of a vertical uniform magnetic field on a two-dimensional flow configuration are studied by Venkatachalappa \& Subbaraya (1993) and Alchaar, Vasseur \& Bilgen (1995). Venkatachalappa \& Subbaraya obtain a strong reduction of the convective flow, where the core is quasi-stagnant and the motion is limited to thin boundary layers by the walls. Alchaar et al. carry out a specific comparison between their numerical result and the analytical model proposed by Garandet, Alboussière \& Moreau (1992), which is based on the assumption of a parallel core flow. They show that the analytical model is in good agreement with the numerical results over a limited parameter range, provided that the enclosure has an aspect ratio larger than 3.

Ozoe \& Okada (1989) report the results of a three-dimensional numerical investigation of the directional effect of a steady magnetic field on side wall convection in a cube of molten silicon $\left(\mathrm{Pr}=5.4 \times 10^{-2}\right)$. They find that the longitudinal orientation of the magnetic field is the most effective for suppressing convection. The transverse 
direction is the least effective which is understandable since Ben Hadid \& Henry (1994) point out that such a field has no effect on a strictly two-dimensional flow field. The results of an experimental study of the flow of molten gallium in a cube are reported in Okada \& Ozoe (1992) where they confirm qualitatively their earlier numerical findings.

A numerical investigation is carried out by Ben Hadid \& Henry (1996) of threedimensional flows of mercury $\left(\mathrm{Pr}=2.6 \times 10^{-2}\right)$ in a cylindrical cavity of aspect ratio 4 , with different orientations of the magnetic field. They find good agreement with the analytical estimates of Garandet et al. (1992) and Alboussière, Garandet \& Moreau $(1993,1996)$ for the damping of the velocity fields. This approach has been extended more recently to a rectangular cavity geometry by Ben Hadid \& Henry (1997), where they include free-surface effects. Interesting changes in the flow structure are reported and these appear to be closely linked to the distribution of the induced currents and their interaction with the applied magnetic field.

Recent experiments by Davoust et al. (1995) focus on the damping of convective flow of mercury in a horizontal cylindrical cavity of aspect ratio 10 by a uniform vertical magnetic field. The structure of the steady flow is investigated at large values of the Hartmann number in the light of current theoretical predictions and good qualitative agreement is found. A study of the time-dependent convective flow is also performed which shows in particular that damping is found to occur for small values of the Hartmann number between 1 and 10.

Most of the numerical and theoretical work reviewed above is concerned with the asymptotic limit of large magnetic fields which may be beyond practical implementation. We have therefore chosen to investigate the flow behaviour over a range of applied magnetic fields and to use the combined strength of numerical and experimental approaches. We have used a transverse field since McKell et al. (1990) showed that moderate fields with this configuration produce interesting dynamical effects. The mathematical model and numerical methods are outlined in $\$ 2$. Brief details of the experimental investigation are given in $\$ 3$ where we also discuss the precautions required when making measurements in the presence of a magnetic field. The numerical and experimental results are discussed in $\$ 4$ where we detail both the spanwise and lengthwise structure of the flow field. Finally we draw some conclusions from our results and speculate on the consequences of them for the more complicated motions found at higher temperature differences.

\section{Mathematical model and numerical techniques}

The numerical model consists of a rectangular cavity of square cross-section filled with gallium, with electrically insulating boundary conditions imposed on all walls. It has aspect ratio $A_{x}=l / h=4$, where $l$ is the length of the cavity and $h$ is its height, as shown schematically in figure 1 .

The endwalls are isothermal and held at $T_{c}$ and $T_{h}$, where the suffixes $c$ and $h$ correspond to cold and hot respectively. Thus, a horizontal temperature gradient is applied and this drives a convective circulation within the cavity.

We will refer to different cross-sections of the cavity, in order to describe both the numerical and the experimental flows. We denote by $X Y-, Y Z$ - and $X Z$-crosssections, the planes which are perpendicular to the $z-, x$ - and $y$-axes respectively and in addition, we indicate the coordinates of their centres. Examples of three crosssections, centred on $x=A_{x} / 2, y=0$ and $z=0$ can be seen in figure 1 . These will commonly be referred to as the central cross-sections. 
The free convection problem is modelled by the Navier-Stokes equations coupled to an energy equation and subject to the Boussinesq approximation (see for instance Braunsfurth et al. 1997). The characteristic scales chosen to non-dimensionalize the equations are $h, v / h, h^{2} / v, \rho v^{2} / h^{2}$ and $\gamma=\left(T_{h}-T_{c}\right) / A_{x}$, where $v$ is the kinematic viscosity and $\rho$ the density of gallium, and they represent respectively length, velocity, time, pressure and temperature.

A horizontal uniform magnetic field, $\boldsymbol{B}=B_{0} \boldsymbol{e}_{B}$, is applied in a direction transverse, i.e. orthogonal, to the applied temperature gradient and gravity. Thus, currents are induced within the cavity which arise from the motion of the conducting fluid through the magnetic field lines. The high electric conductivity of the material ensures that the induced magnetic field associated with these currents is negligible, so that the externally applied magnetic flux density remains uniform. Also, interaction between the induced electric currents and the applied field results in a magnetic body force, called the Lorentz force, which is given by

$$
\boldsymbol{F}_{L}=\boldsymbol{j}_{d} \times \boldsymbol{B},
$$

where $\boldsymbol{j}_{d}$ is the dimensional electric current density and $\boldsymbol{B}$ the magnetic flux density. The electric current density, normalized by $\left(\sigma v B_{0}\right) / h$ where $\sigma$ is the electric conductivity of the fluid, is defined by Ohm's law in a moving frame of reference to be,

$$
\boldsymbol{j}=-\nabla \Phi+\boldsymbol{u} \times \boldsymbol{e}_{B},
$$

where $\boldsymbol{j}$ is the dimensionless electric current density, $\Phi$ is the induced electric potential and $\boldsymbol{u}=(u, v, w)$ is the fluid velocity. Furthermore, $\boldsymbol{j}$ satisfies the continuity equation,

$$
\nabla \cdot \boldsymbol{j}=\mathbf{0} .
$$

By combining equations (2.2) and (2.3) we obtain

$$
\nabla^{2} \Phi=\boldsymbol{e}_{B} \cdot(\nabla \times \boldsymbol{u}) .
$$

Thus, the resulting non-dimensional equations of motion are the continuity equation, the equations for the transport of momentum, the equation for the transport of energy and equation (2.4). The following boundary conditions are imposed:

$$
\begin{gathered}
\partial T / \partial y=0 \text { on } y= \pm 1 / 2, \quad \text { and } \partial T / \partial z=0 \quad \text { on } z= \pm 1 / 2, \\
T=0 \text { on } x=0, \quad \text { and } T=A_{x} \quad \text { on } \quad x=A_{x},
\end{gathered}
$$

where $T$ is the reduced temperature, and

$$
\boldsymbol{u}=\mathbf{0} \text { and } \partial \Phi / \partial n=0 \text { on all boundaries. }
$$

Under these conditions, the values of the electric potential and the pressure are not unique. Thus, their absolute value is set to zero at a convenient point within the cavity.

The scaling of the governing equations naturally leads to expressions for the Grashof number, $G r=\beta \gamma g h^{3} / v^{2}$, the Hartmann number, $H a=B_{0} h(\sigma / v \rho)^{1 / 2}$ and the Prandtl number, $\operatorname{Pr}=v / \kappa$, where $\beta$ is the coefficient of thermal expansivity and $\kappa$ the thermal diffusivity.

It is useful to recall that the three-dimensional equations of motion associated with the above cited boundary conditions are invariant under the following transformations:

(i) $\left(x-A_{x} / 2, y, z\right) \rightarrow\left(-\left(x-A_{x} / 2\right), y,-z\right)$,

(ii) $\left(x-A_{x} / 2, y, z\right) \rightarrow\left(x-A_{x} / 2,-y, z\right)$. 
The first of these two $\boldsymbol{Z}_{2}$ symmetries is referred to as centro-symmetry by Gill (1966) and it expresses the fact that the flow is unchanged by a rotation of $\pi$ about the $y$-axis in the centre of the enclosure. It is an intrinsic property of the Boussinesq flow, as the buoyancy term is taken to be linearly dependent on temperature and it is unchanged by the addition of a magnetic field. The second transformation describes a reflectional symmetry commonly referred to as left-right symmetry in convection problems.

The governing equations were computed on a three-dimensional domain using a spectral element method where the spatial discretization has been carried out using $31 \times 15 \times 15$ Gauss-Lobatto-Legendre collocation points. A detailed description of the methods and their implementation in MHD convection flows can be found in Ben Hadid \& Henry (1997). They report that the grid we have used is sufficiently accurate for confined MHD flow and the interested reader is referred to their paper for a detailed discussion of numerical accuracy tests.

We now consider a two-dimensional version of the convective flow such that motion is restricted to the two directions $x$ and $z$, while a magnetic field is applied along the $y$-direction. It was shown explicitly by Ben Hadid \& Henry (1994) that the magnetic field applied in the transverse direction will have no effect on the flow.

In this case, the magnetic equation (2.4) can be re-written as

$$
\nabla^{2} \Phi=\frac{\partial w}{\partial x}-\frac{\partial u}{\partial z},
$$

since $\boldsymbol{B}_{0}$ is applied along the $y$-direction. In a two-dimensional flow, the velocity can be expressed in terms of a stream function $\Psi$ by setting

$$
w=-\frac{\partial \Psi}{\partial x}, \quad u=\frac{\partial \Psi}{\partial z},
$$

which yields

$$
\nabla^{2}(\Phi-\Psi)=0 .
$$

When the boundary conditions $\partial \Phi / \partial n=0$ and $\partial \Psi / \partial n=0$ are applied this yields the solution

$$
\Phi-\Psi=\text { constant }
$$

Hence, the induced electric potential is the exact image of the stream function. The electric current is given by (2.2) and since $\boldsymbol{u} \times \boldsymbol{e}_{B}=\boldsymbol{u} \times \boldsymbol{y}=\nabla \Psi$,

$$
\boldsymbol{j}=-\nabla \Phi+\nabla \Psi=0 .
$$

Thus, a two-dimensional convective flow is unaffected by the action of a uniform magnetic field applied perpendicular to the plane of a cavity with electrically insulating boundaries since the magnetic body force is zero. Hence, all calculations reported here were performed on the full three-dimensional problem.

\section{The experiment}

\subsection{Experimental apparatus}

A schematic diagram of the apparatus is shown in figure 2. It is described in detail in Braunsfurth \& Mullin (1996) and thus we will only give a brief description of the points pertinent to the present study.

The working section of the experiment consisted of an insulating rectangular channel which held the liquid gallium between two thermally conducting plates. 


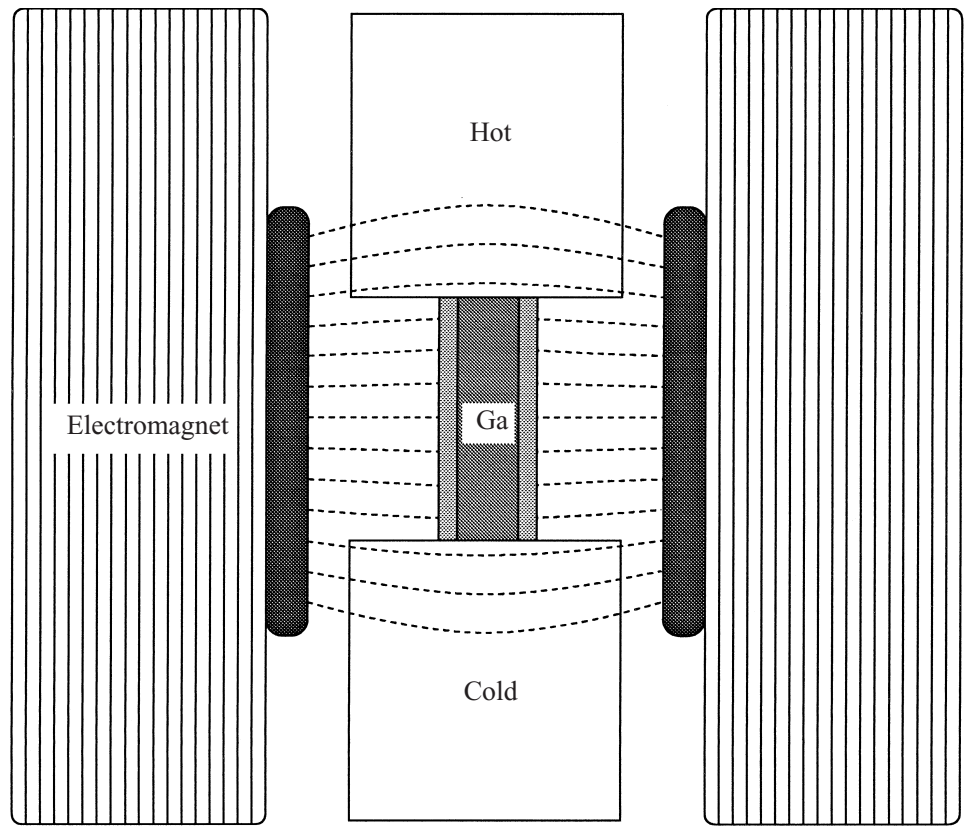

FIGURE 2. Schematic diagram of the experimental setup viewed from above.

These were made from a $1 \mathrm{~mm}$ thick molybdenum sheet, which is a good conductor and is also impervious to attack by gallium. Each endwall was the side of a copper box of capacity 0.71 , containing silicone oil, whose temperature was held constant to within $\pm 0.05^{\circ} \mathrm{C}$ by a commercial temperature controller. The experiment was further enclosed in an air cabinet whose temperature was held constant at $32 \pm 0.5^{\circ} \mathrm{C}$. In particular, these precautions ensured that good uniformity and stability of the applied temperature at the two endwalls of the container was achieved. The channel containing the gallium was thermally insulating and was made from a machinable ceramic called pyrophelite. Its thermal conductivity is 29 times smaller than that of gallium and it is also electrically insulating. A non-conducting ceramic lid was fitted on top of the gallium to form the upper boundary. Hence the four faces of the enclosure were insulating while the endwalls were conducting.

The channel containing the gallium was centred between the 4 in. pole pieces of an electromagnet, allowing steady fields of up to $1250 \mathrm{G}$ to be reached using a $30 \mathrm{~V}$, 3 A stabilized d.c. power supply. Thus, Hartmann numbers in the range 0 to 64 could be obtained. The strength of the applied magnetic field was controlled manually by regulating the electric current supplied to the coils of the electromagnet. The current range was divided into steps of $0.2 \mathrm{~A}$ and the magnetic field was calibrated against the applied current using a transverse probe Gaussmeter. Both positive and negative magnetic fields were applied, as their effect on the flow was found to be identical. The hysteresis due to the magnetization of the soft iron poles was negligible and hence was ignored for these measurements. A residual field of approximately $20 \mathrm{G}$ was measured. This corresponds to a Hartmann number of 1 and thus the uncertainty on the absolute value of the Hartmann number was \pm 1 .

In addition, in order to obtain zero values of the Hartmann number for the study of the flow in the absence of a magnetic field, the residual field was eliminated with a 
small reverse current, so that the layer of gallium was subjected to a resulting field of less than $1 \mathrm{G}$. This corresponded to a value of the Hartmann number of $H a=0 \pm 0.1$, which was found to have a negligible effect on the flow, as will be seen in $\S 4$.

The spatial distribution of the magnetic field within the cavity was measured for an applied d.c. current of $3 \mathrm{~A}$, which corresponded to the largest magnetic field that could be applied with the present system. The measurements were carried out using a Gaussmeter with a Hall-effect probe. This was held in a micro-manipulator and moved manually along a $Y Z$-cross-section of the cavity on a grid of $4 \times 4$ measurement points. Measurements were taken on two $Y Z$-cross-sections at $x=2.0$ and $x=3.6$. The magnetic field was found to be uniform within $\pm 0.3 \%$ of its mean value on each of these two cross-sections. Further measurements were taken on the $X Y$-plane at the bottom of the cavity. Here, the field was found to be uniform within $\pm 1.5 \%$ of the measured mean value. In addition, these measurements were repeated for an applied current of $1 \mathrm{~A}$ and similar results were obtained. The very good uniformity that was achieved justifies the choice of an electromagnet with large polar pieces and rules out the occurrence of effects due to non-uniformities in the magnetic field such as those studied by Neubrand et al. (1995).

\subsection{Experimental measurements}

Temperature was measured with a type $\mathrm{K}$ insulated thermocouple of diameter $0.25 \mathrm{~mm}$ which was accurately positioned within the melt using a micromanipulator. The precision achieved in the measurement of relative temperatures was better than $\pm 0.01^{\circ} \mathrm{C}$. Care was taken to ensure that the probe did not perturb the convective flow and the experimental procedure was very similar to that reported by Braunsfurth et al. (1997). Thus, we will only recall the essential points here.

The vertical temperature difference $\Theta$, measured at a given location in the cavity, is of particular interest since it reflects the amount of heat transferred by convection. If the liquid gallium in the cavity were replaced by a solid, heat transport would take place by conduction alone. The temperature would then simply be a function of lengthwise position $x$, and there would be no vertical temperature dependence. Here, heat transport can take place by fluid convection as well as conduction. However, this convective heat flow is weak and is associated with motion of hot liquid in the top half of the container, progressing from the hot end to the cold, and vice versa in the bottom half.

In order to obtain estimates of $\Theta$, vertical temperature profiles were recorded in the following way. Measurements were taken at 20 different vertical positions, at $x=2.0$ and $y=0$. At each point the flow was allowed $60 \mathrm{~s}$ to settle from the disturbances caused by the movement of the probe. Then a sample of 600 data points was taken over a period of $60 \mathrm{~s}$, and the mean, minimum and maximum were recorded. The minimum and maximum gave an estimate of the size of the fluctuations present in the experiment and of measurement noise, and in the present case of steady flow, indicated the size of the error bars on the measurement points. An example of a vertical temperature profile recorded at $x=2.9$ and $y=0$ is displayed in figure 3. It reflects the stable temperature stratification within the cavity due to the buoyancy-driven convective circulation. The vertical temperature difference was then determined by measuring the total amplitude of the profile. In practice there is an unknown offset in all the measurements due to an arbitrary level shift introduced by the electronics. Hence, the zero of the temperature axis was defined to be at the mid-height of the gallium sample, as shown in figure 3 .

Temperature distributions on cross-sections of the cavity orthogonal either to the $x$-axis or the $y$-axis were also measured by recording series of vertical temperature 


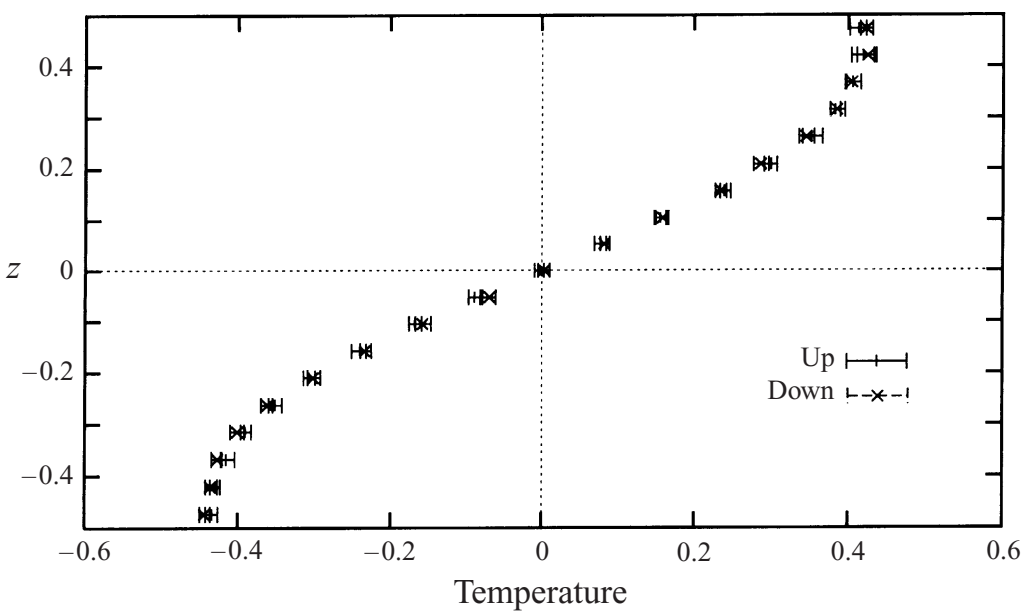

FIGURE 3. An example of an experimentally determined vertical temperature profile. The parameter values were set to $\mathrm{Gr}=4.5 \times 10^{4}, \mathrm{Pr}=2.5 \times 10^{-2}$ and $\mathrm{Ha}=0$.

profiles on a grid pattern. In the case of the $Y Z$-cross-section, the measurements were taken on a grid of 12 transverse points by 20 vertical ones. The probe was initially placed at the bottom of the cavity along a sidewall. It was then moved upwards in steps of $0.7 \mathrm{~mm}$. At each measuring station the flow was allowed to settle for $180 \mathrm{~s}$ before a 3000 records long time series was sampled over a period of $10 \mathrm{~min}$. The mean, minimum and maximum values were retained similarly to the recording of the vertical temperature profiles. When the probe reached the surface of the gallium it was moved transversally by $1.09 \mathrm{~mm}$ and then lowered back down to the bottom of the cavity and the procedure repeated for the 11 remaining transverse positions. All the transverse measurements needed to be carried out without a lid for practical reasons. As discussed by Braunsfurth et al. (1997) profiles are insensitive to the presence of a lid and we believe that this has minimal effect on the flow field. For the central $X Z$-cross-section, temperature was measured on a grid of 26 horizontal points by 16 vertical ones, following a procedure similar to the above, and a lid was present on top of the gallium.

The study of the magnetohydrodynamic flow required additional precautions. As discussed by Davoust (1996), the immersion of a small insulating object, such as the thermocouple probe, into the layer of gallium results in the creation of parallel layers within the core flow. The disturbance to the flow is determined by estimating their thickness, which is of the order of the diameter of the probe over the square root of the local Hartmann number, based on this same characteristic length scale. For $H a=10$ it is less than $6 \%$ of the total depth of the layer and for $H a=60$ it is less than $2 \%$, which suggests that their effect on the flow is insignificant.

It is also worth recalling that in the experiment, the endwalls are electrically conducting. In the presence of a transverse magnetic field, thin parallel layers are expected to form along these walls but we do not expect them to influence the core flow. As will be seen below, the agreement between experimental measurements and numerical simulations performed for electrically insulating walls support these assumptions. In addition, good uniformity of the temperature distribution on the endwalls was found by Braunsfurth et al. (1997) and thus thermo-electric effects between the gallium and the molybdenum are also assumed to be unimportant. 


\subsection{Material properties}

The accurate determination of the absolute value of the experimental parameters relies on precise knowledge of the material properties. This is especially true if we wish to make comparisons with the numerical calculations. The data on the physical properties of molten gallium are sparse and their dependence on temperature is not well known. The available data were collected from a number of sources and discussed by Braunsfurth et al. (1997). In particular, they showed that the value of the Prandtl number is known to within a systematic uncertainty of $16 \%$ which is essentially due to lack of knowledge of the thermal conductivity. However, this is not a major issue here since it has been shown by Juel et al. (1998) that the steady convective flow, which is the focus of this study, is qualitatively the same in the absence of a magnetic field over the entire range of Prandtl numbers studied. Also, this systematic uncertainty is of little importance when comparing one set of experimental measurements with another, as the reproducibility of these results over a period of several months suggests that the sample of gallium retains its purity. However, it should be recalled that variations in $\operatorname{Pr}$ can have an effect on the observed dynamical motion as reported by Braunsfurth $\&$ Mullin (1996) and this remains a topic of further research.

\section{Results: convection in the presence of a transverse uniform magnetic field}

\subsection{Damping of the convective circulation}

A quantitative comparison between experimental results and numerical simulations was performed using measurements of the strength of the convective flow. As discussed in $\S 3$, the vertical temperature difference in the centre of the cavity (at $x=2.0$ and $y=0$ ) was chosen since it is a good indicator of the convective heat transfer. The dependence of the vertical temperature difference on the applied magnetic field was determined experimentally by recording vertical temperature profiles at ten successive fixed values of the Hartmann number ranging from zero up to $H a=64$. The Grashof number was held fixed at $4.6 \times 10^{4}$ and the Prandtl number was $2.5 \times 10^{-2}$. These particular parameter values were chosen so that the flow was steady and of sufficient strength to provide a good quality signal. The extracted experimental values of $\Theta$ were compared with three-dimensional numerical data calculated for $G r=5.0 \times 10^{4}$ and $\operatorname{Pr}=3.0 \times 10^{-2}$. These numerical parameter values were chosen as a matter of convenience since the absolute experimental conditions were not known precisely at the time the calculations were performed. As will be seen below, the agreement between experimental and numerical results is very satisfactory and we judged it unnecessary to repeat the very expensive calculations at the precise experimental parameter values.

The results are plotted in figure 4. It can be seen that the vertical temperature difference is a monotonically decreasing function of $\mathrm{Ha}$ and thus the amount of heat transferred by convection is reduced as the magnetic field is increased. Hence the applied magnetic field damps the convective circulation as found in other investigations. The correspondence between the experimental and numerical results is very good which is highly satisfactory given the uncertainty in the experimental parameters. For sufficiently high Hartmann numbers, they both tend to follow the same scaling law. However, the value of the scaling coefficient cannot be related to any theoretical ones since we were unable to apply a sufficiently large magnetic field to approach an asymptotic state. Thus, in order to investigate this regime, numerical calculations were carried out beyond the experimental range of Hartmann numbers so that comparison 


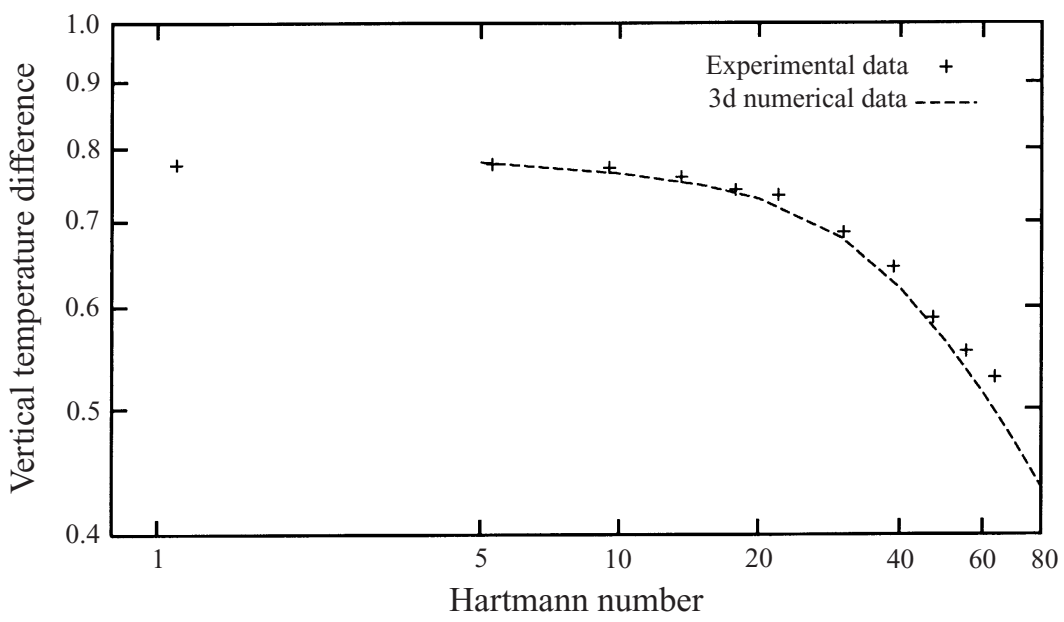

FIGURE 4. Dependence of the vertical temperature difference on the Hartmann number: comparison between experimental results and those of three-dimensional numerical calculations.

could be made with the theoretical predictions. We found that $\Theta$ and similarly the maximum horizontal and vertical velocities in the central $X Z$-plane, $u_{\max }$ and $w_{\max }$, vary approximately as $\mathrm{Ha}^{-1}$ for $\mathrm{Ha}>100$. These results are in agreement with those obtained by Ben Hadid \& Henry (1996) in a cylindrical cavity and confirm the results of Alboussière, Garandet \& Moreau (1996).

The damping of the convective circulation can be understood in terms of the induced electric currents and potentials. The magnetic body force is given by $\boldsymbol{F}=$ $H a^{2} \boldsymbol{j} \times \boldsymbol{e}_{B}$, where $\boldsymbol{j}$ represents the electric currents that arise due to the interaction between flow and magnetic field. The currents have two distinct sources, $\boldsymbol{u} \times \boldsymbol{e}_{B}$ due to movement of the fluid through the magnetic flux lines, and $-\nabla \Phi$, the electric field which results from this reorganization and counteracts the former term. These two contributions cancel in a purely two-dimensional configuration as discussed in $\$ 2$. In the present case, the distribution of electric currents is largely influenced by the strong gradients of electric potential induced on the sidewalls where velocity is zero. In the central part of the cavity, there is a weak induced current density which is essentially vertical since $u$ is the dominant component of the velocity there. Vectors of induced current density which were obtained for numerical calculations performed for $H a=100$ are plotted in figure 5(a) on the central $X Z$-plane. It can be seen that they are strictly vertical in the central section of the cavity. This, in turn, results in a magnetic body force which damps the flow. The potential iso-lines (figure $5(b)$ ) reflect the streamlines of the convective flow.

\subsection{Alteration of the flow structure}

A distinct advantage of numerical simulations is that they can be used to provide a full and detailed picture of the model flow. This is particularly beneficial in the present case given that we have established good agreement between calculations and experiment. We therefore used the numerical results to investigate the modification of the flow structure by the magnetic field and then the global effects were demonstrated clearly in the experiment. All the numerical results presented below were calculated for $G r=5.0 \times 10^{4}$ and $P r=3.0 \times 10^{-2}$.

Similar agreement was found between experiment and three-dimensional model 
(a)

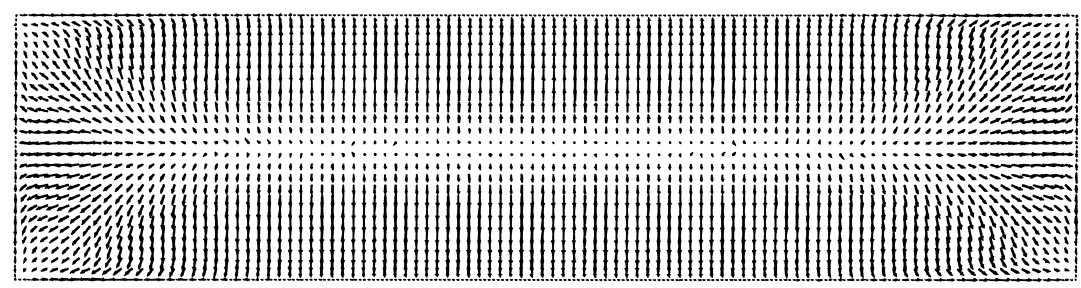

(b)

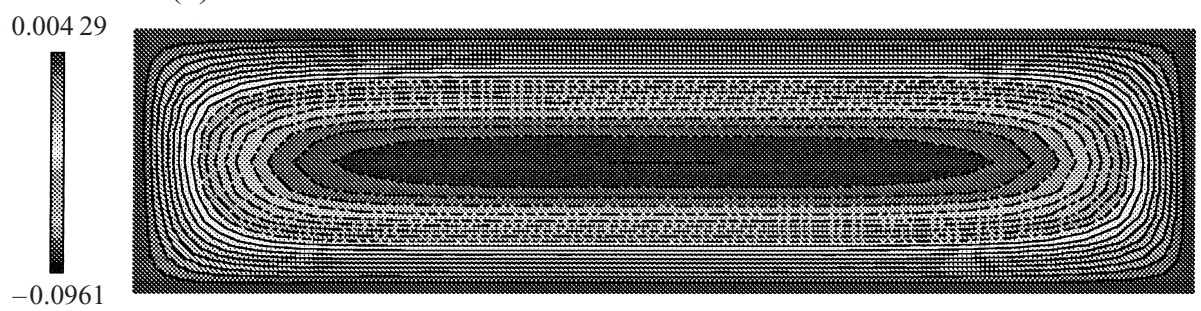

FIGURE 5. Projection of current density vectors $(a)$ and plot of electric potential iso-lines $(b)$ for $G r=5.0 \times 10^{4}, \operatorname{Pr}=3.0 \times 10^{-2}$ and $H a=100$ on the central $X Z$-plane.

(a)

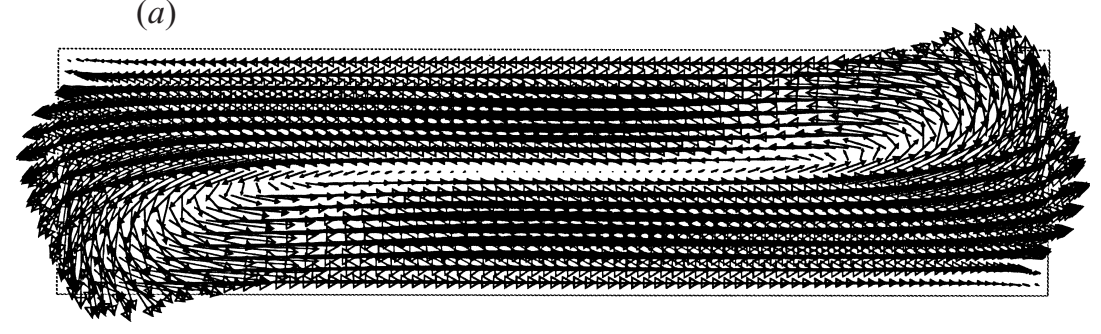

(b)

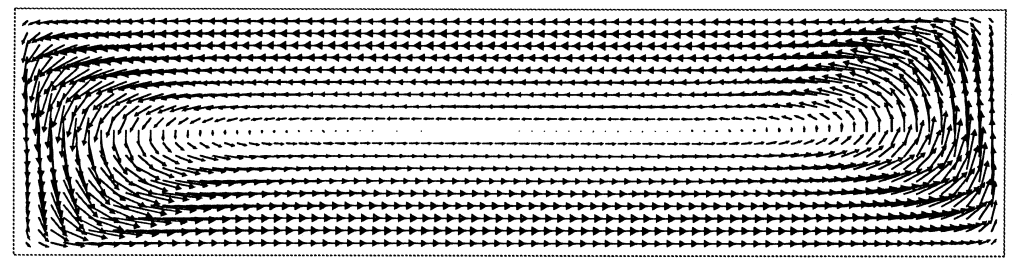

FIGURE 6. Plot of numerical velocity vectors, calculated for $G r=5.0 \times 10^{4}$ and $P r=3.0 \times 10^{-2}$, on the central $X Z$-plane: (a) $H a=0,(b) H a=100$.

in the absence of a magnetic field and we refer to Juel et al. (1998) for a detailed discussion of the structure of the free convective flow. In the following, we will only recall the features which are essential to the present comparison.

\subsubsection{Lengthwise structure of the flow}

In figures $6(a)$ and $6(b)$, velocity vectors, which were calculated for $H a=0$ and $H a=100$ respectively, are plotted on the central $X Z$-plane. The same scale was chosen to represent both flows and as a result, the projection of velocity vectors of 
figure $6(a)$ is such that they jut over the boundaries. It is clear from this picture that the velocity field is strongly reduced in the presence of a magnetic field. At $H a=0$, the maximum velocity is $u_{\max }=1.13$ while for $H a=100, u_{\max }=3.45 \times 10^{-1}$. However, it can also be seen that the structure of the flow is strongly modified. In figure 6(a), dark structures can be seen in the central part of the cavity. They reflect the presence of three-dimensional secondary flows, which arise from the interaction between the recirculation flows of the two end regions, as described by Juel et al. (1998). This interaction takes place in the centre of the cavity. Thus, a threedimensional recirculation structure is generated, which is found to be at the origin of the destabilization of the flow, at larger values of the Grashof number. At $H a=100$, it can be seen that the central part of the flow is parallel and that the role of the end regions is simply to recirculate the fluid within the cavity. Thus, the structure of the flow is altered in the presence of a strong magnetic field, so that it becomes increasingly uniform over the length of the cavity.

The lengthwise structure of the temperature field was investigated experimentally by recording temperature distributions along the central $X Z$-plane for two values of the Hartmann number $H a=0$ and $H a=64$, following the procedure described in $\S 3$. A relatively large Grashof number of $1.3 \times 10^{5}$ was chosen in order to ensure a relatively large amount of convection and hence obtain significant curvature of the isotherms. As a result, we expected to observe a bigger effect of the magnetic field on the temperature field in this case. The Prandtl number was set to $\mathrm{Pr}=2.3 \times 10^{-2}$. Since the measured temperature values were relative, a zero temperature reference point was chosen close to the cold endwall, where the temperature was lowest. Temperature contours, calculated from the recorded data using cubic interpolation, are displayed in figure 7(a) for $\mathrm{Ha}=0$ and in figure 7(b) for $\mathrm{Ha}=64$. At these parameter settings the flow is time-dependent. This is evident in its general irregular appearance which results from averaging the unsteady flow, as discussed in $\$ 3$. Here, each point was averaged for $10 \mathrm{~min}$ which we estimated to be long enough to capture the essential elements of the convective flow. In figure $7(b)$, the isotherms are smooth over the entire domain, which suggests that the magnetic field has a stabilizing effect on the flow, as previously found by Hurle, Jakeman \& Johnson (1974) and Davoust et al. (1995). Furthermore, the curvature of the experimental contours is reduced between figures $7(a)$ and $7(b)$ suggesting an overall damping of the convective heat flow. In particular, it can be seen that the spacing between the isotherms is more uniform for $H a=64$ than for $H a=0$. This suggests that an alteration of the lengthwise structure of the experimental flow takes place when a transverse uniform magnetic field is applied, in accordance with the numerical findings.

A closer inspection of the data confirmed the above conclusion. Lengthwise profiles at $z=0.49$ and $z=-0.49$ were extracted from the series of measurements and the resulting profiles are displayed in figure $8(a)$ for $H a=0$ and $8(b)$ for $H a=64$. For each profile, the mean, maximum and minimum values have been interpolated linearly in order to highlight the regions of oscillations. At $\mathrm{Ha}=0$, the strongest oscillations are observed in the profile at $z=0.49$ towards the cold end. These are indicated by a splitting of the maximum and minimum values in the left-hand portion of figure 8(a). At $\mathrm{Ha}=64$, the oscillations have been damped in the bulk of the material, although they are not entirely inhibited near the upper surface, as seen in figure $8(b)$. In addition both profiles are more symmetrically disposed in the presence of a magnetic field. This symmetry is also found in the three-dimensional numerical model of free convection, which is centro-symmetric.

A more detailed investigation of the alteration of the experimental flow by the 
(a)

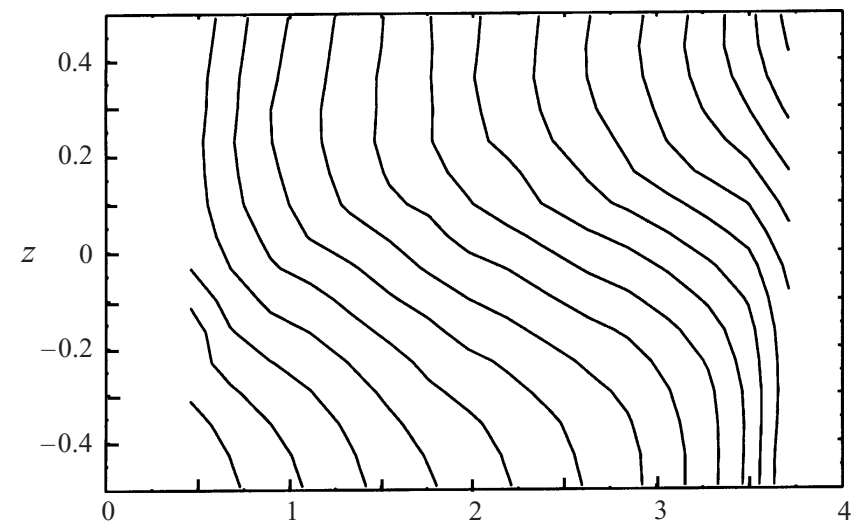

(b)

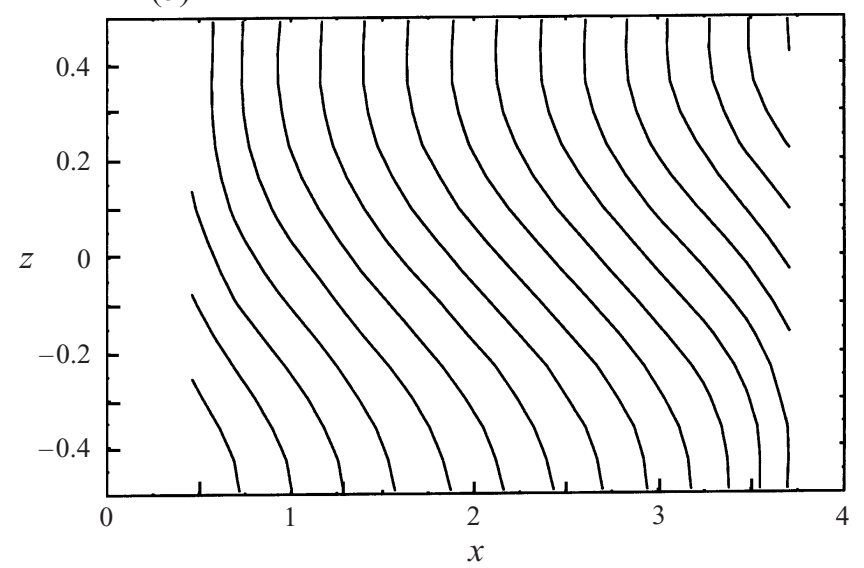

FigURE 7. Experimental temperature contours plotted on the central $X Z$-plane: $(a) H a=0,(b)$ $H a=64$. The measurements were taken for $G r=1.3 \times 10^{5}$ and $P r=2.3 \times 10^{-2}$. In each plot, the contour situated furthest to the left corresponds to a relative non-dimensional temperature of 0.2 . Also, the temperature variation between two neighbouring contours is of 0.2. Thus, the contour located furthest to the right in $(a)$ corresponds to a temperature of 3.4 and in $(b)$ of 3.6. However, it is also worth recalling that the experimentally determined temperatures values are relative, and that the zero of the temperature axis was defined to be at the bottom measurement point situated closest to the cold endwall, where the lowest value was measured.

magnetic field was conducted by recording vertical profiles for $H a=0$ and $H a=64$ at different lengthwise positions. All the measurements were taken halfway along the width of the cavity following the procedure described in $\S 3$. Results are presented in figures $9(a), 9(b)$ and $9(c)$ of profiles sampled at $x=1.0, x=2.0$ and $x=$ 3.0 respectively. In each plot, comparison is made between the measurements with and without magnetic field. In all three cases, the reduction of the temperature stratification, which takes place when the magnetic field is applied, is different in the top half of the cavity to that in the bottom part. This is particularly obvious in figure $9(a)$ where the damping is concentrated in the bottom half while the upper part of the temperature profile remains almost unaffected. Due to the centro-symmetric properties of the system, the vertical profiles are generally asymmetric about $z=0$ except at $x=x_{c}$, which is the centre of the circulation in the convective flow and 


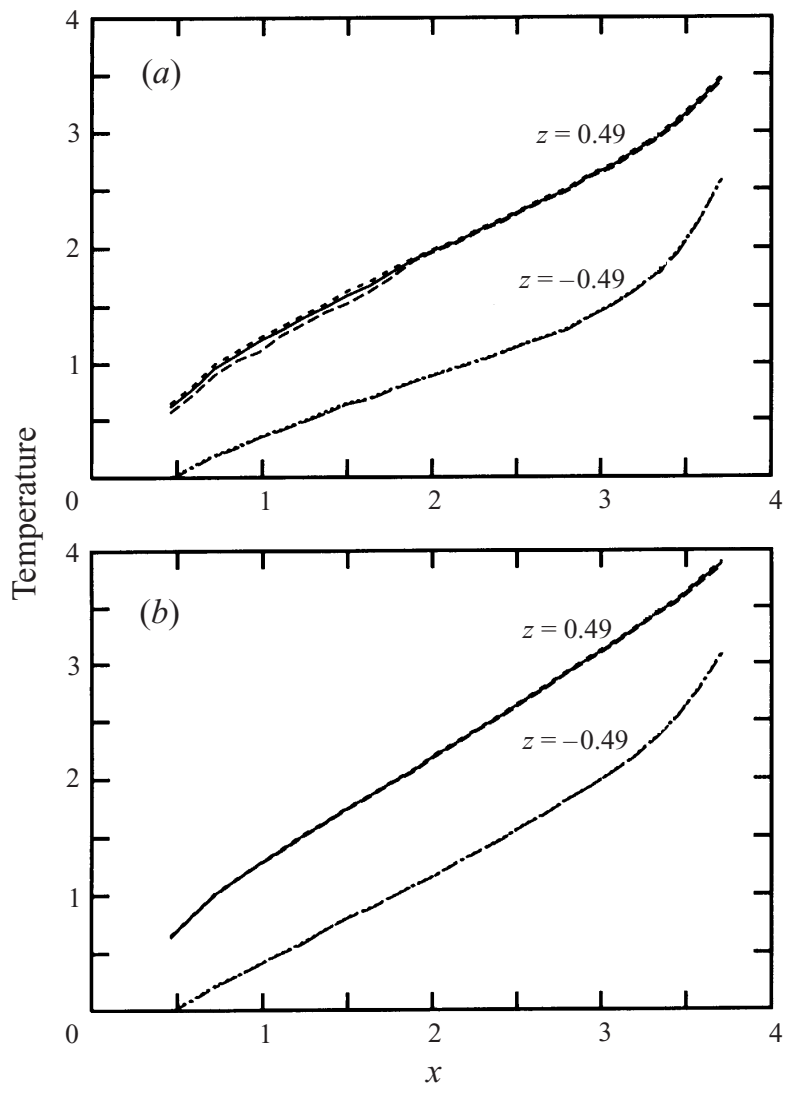

Figure 8. Experimental lengthwise profiles at $z=0.49$ and $z=-0.49$ : (a) $H a=0$, (b) $H a=64$.

is situated at $x_{c}=2.0$ in the numerical model. However, in the experiment, Juel et al. (1998) find that the maximum vertical temperature difference $\Theta$ is located at approximately $x_{c}=3.0$ and thus that the centre of the convective circulation is displaced towards the hot end of the cavity. The reasons for this discrepancy are not at present understood, although it is thought that a Boussinesq approximation is inadequate to model the experimental flow since the material properties of gallium are strongly temperature dependent. Thus, the amplitude of the top half of the profiles taken for $x>x_{c}$ is larger than the bottom part and vice versa for $x<x_{c}$. It is interesting to note that the asymmetric half of each profile, which has the largest amplitude, is consistently more reduced by the magnetic field. Hence, in figure $9(b)$ for $x=2.0$, the bottom half of the profile is damped more heavily than the top part and vice versa in figure $9(c)$ which is situated slightly beyond the centre of the experimental circulation. Overall, the results displayed in figures $9(a), 9(b)$ and $9(c)$ indicate that the presence of a sufficiently large magnetic field promotes symmetry about $z=0$ in the experimental profiles and thus results in a more uniform flow over the lengthwise extension of the cavity. These results confirm the global observations made on the temperature contours displayed in figure 7.

Finally, the dependences of the vertical temperature difference on the lengthwise position $x$ at $H a=0$ and $H a=64$ were compared in figure 10 in order to provide a quantitative measurement of the effect of the magnetic field on the lengthwise asymmetry of the flow. Each value of $\Theta$ was obtained using the procedure described 

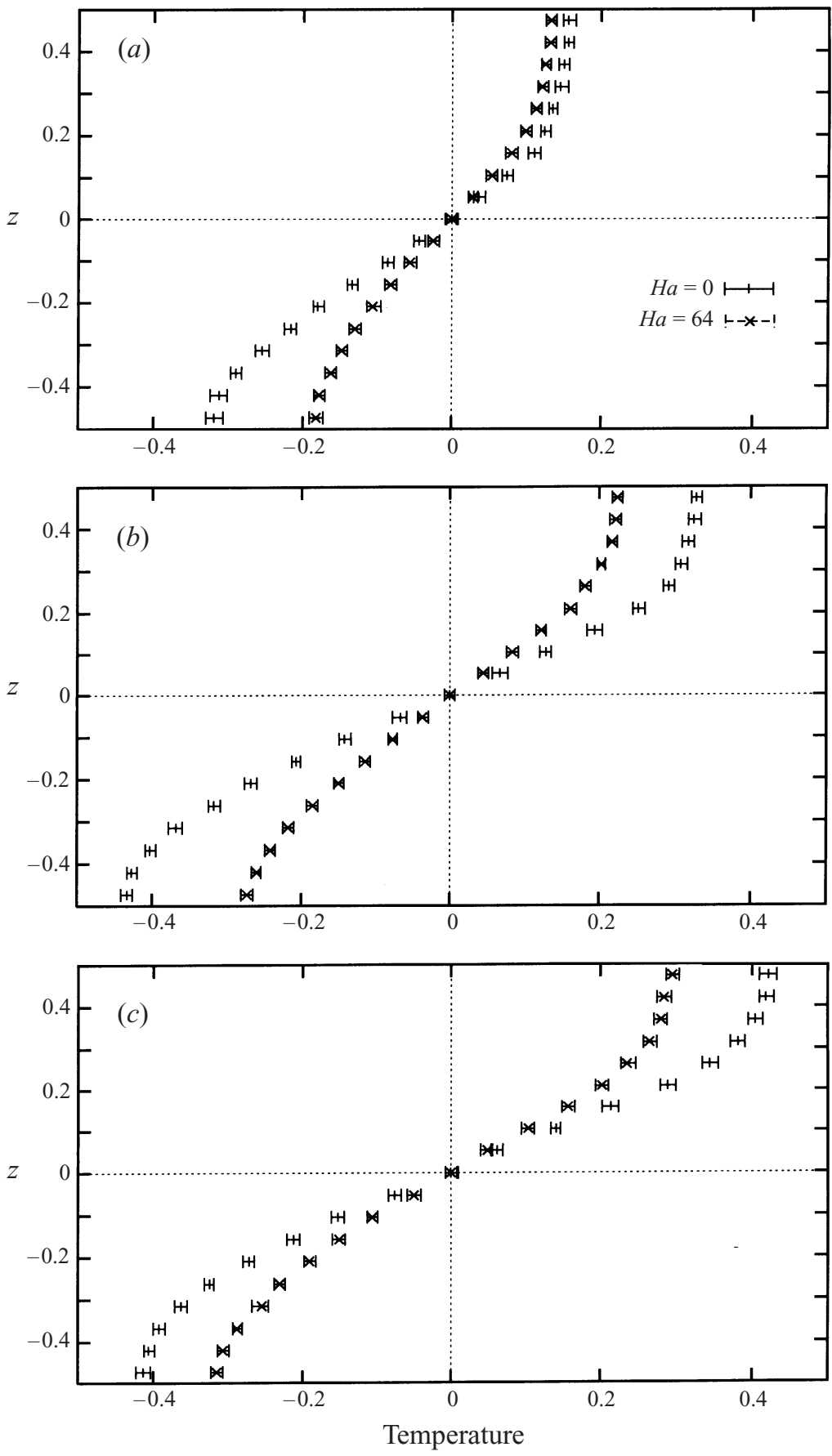

FIGURE 9. Comparison between experimental vertical temperature profiles sampled for $H a=0$ and $H a=64$. The measurements were made at different lengthwise locations for $\mathrm{Gr}=4.6 \times 10^{4}$ and $\operatorname{Pr}=2.5 \times 10^{-2}:(a) x=1.0,(b) x=2.0,(c) x=3.0$. 


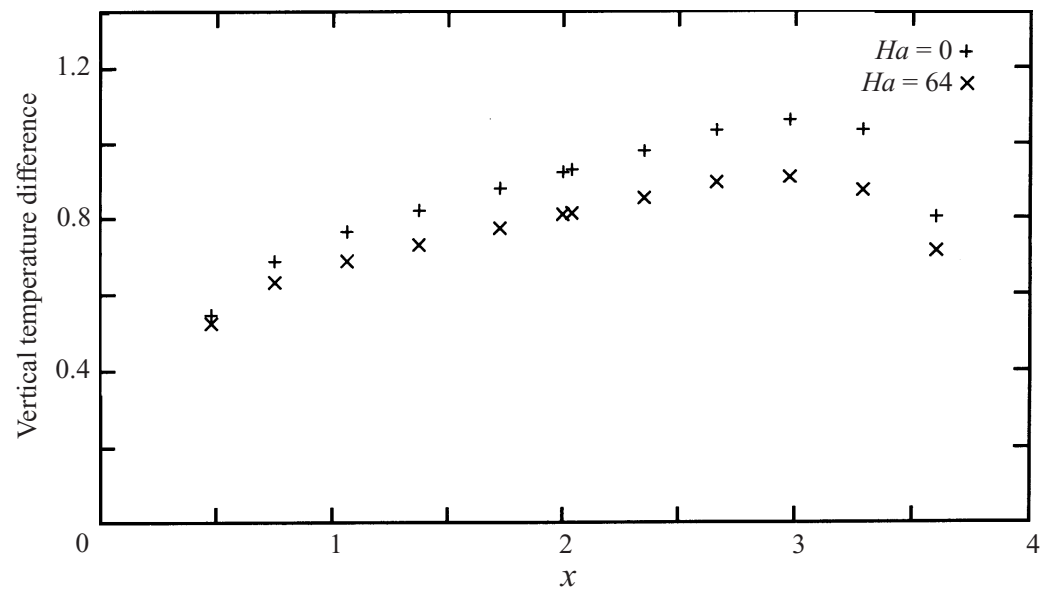

FIGURE 10. Comparison between the experimental dependence of the vertical temperature difference on the lengthwise position $x$ at $H a=0$ and $H a=64$. The measurements were made for $G r=9.8 \times 10^{5}$ and $\operatorname{Pr}=2.4 \times 10^{-2}$.

in §3. It is clear from figure 10 that damping is most efficient around $x=3.0$ where the convective flow is the strongest. The effect increases monotonically and quasi-linearly with $x$ and is approximately $27 \%$ weaker at $x=2.0$ and $50 \%$ weaker at $x=1.0$. These findings clearly suggest that the asymmetry of the experimental flow is reduced in the presence of a transverse uniform magnetic field. However, it can also be seen in figure 10 that the centre of the circulation in the experimental flow is not relocated for $H a=64$. Thus, it appears that the reduction in amplitude of the experimental asymmetry is simply a consequence of the increased uniformity of the flow in the presence of the magnetic field.

\subsubsection{Spanwise structure of the flow}

The spanwise structure of the flow was first examined numerically in a similar way to the study reported in $\S 4.2 .1$. An investigation was carried out into the effect of an applied magnetic field on the velocity components and vertical temperature difference, which was calculated for the spatial location $x=2.0, y=0$. The results are displayed as a function of $\mathrm{Ha}$ in figure 11. It can be seen that the vertical temperature difference $\Theta$ varies with $H a$ in a similar manner to the maximum longitudinal and vertical components of the velocity, $u_{\max }$ and $w_{\max }$. In the present case of a uniform transverse magnetic field, the maximum value of the longitudinal velocity, $u_{\max }$, is found within the core flow and its asymptotic variation is in agreement with the theoretical predictions of Alboussière, Garandet \& Moreau (1996). The maximum value of the transverse component of the velocity, $v_{\max }$, is smaller than that of the three other quantities at $\mathrm{Ha}=0$. This is because the transverse direction $y$ is not a main direction of flow. The main buoyancy-driven circulation is essentially twodimensional, as discussed by Braunsfurth et al. (1997), although secondary, transverse cross-flows exist at all values of the Grashof number. It is interesting to note that $v_{\max }$ undergoes an initially strong reduction at intermediate values of the Hartmann number but varies asymptotically as $\mathrm{Ha}^{-1}$. This indicates that the presence of a moderate uniform transverse magnetic field has the effect of restricting the flow to the two directions $x$ and $z$ and hence favours two-dimensional motion. These results 


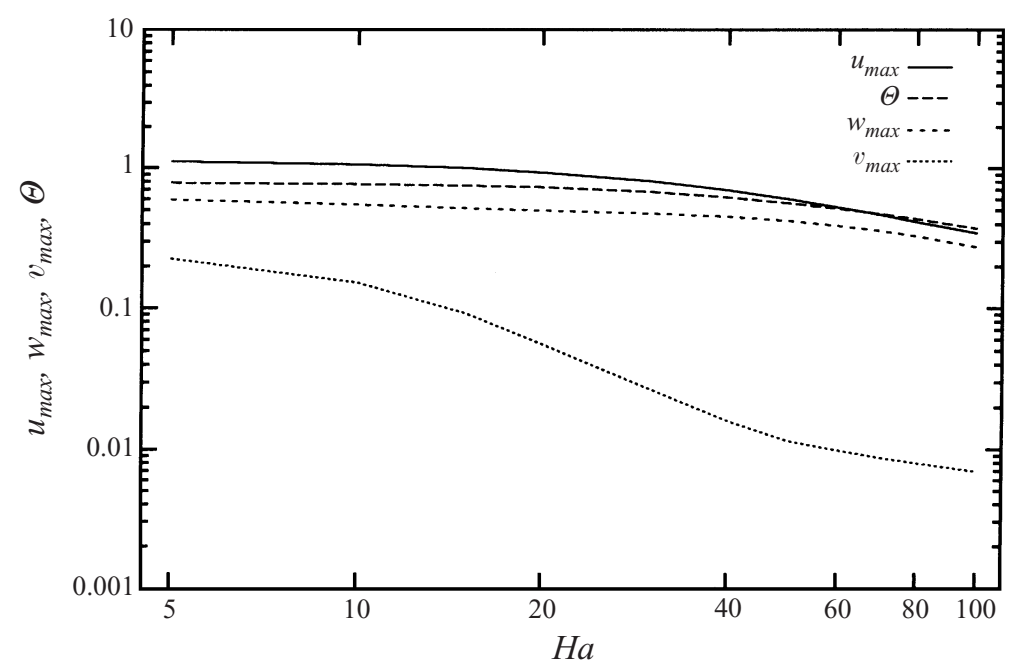

FIGURE 11 . Dependence of the maximum velocity components $\left(u_{\max }, v_{\max }\right.$ and $\left.w_{\max }\right)$ and of the numerical vertical temperature difference $(\Theta)$ on the Hartmann number.

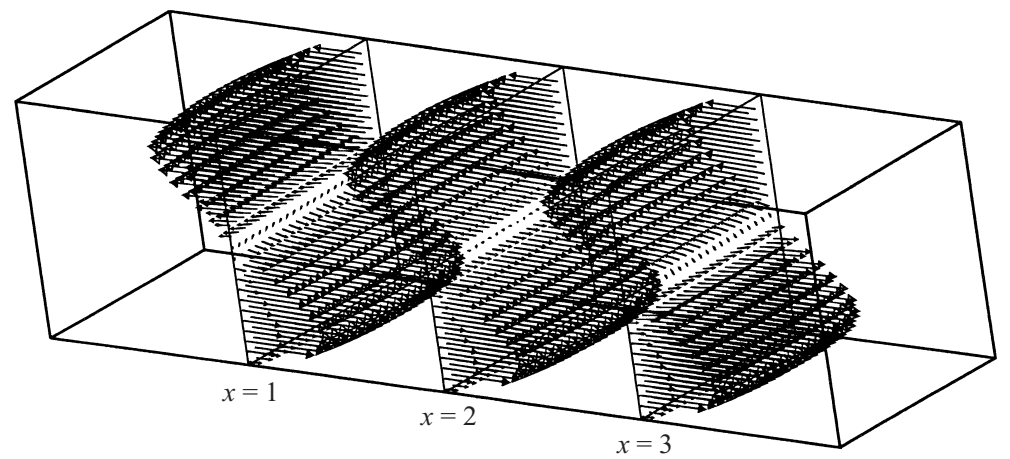

FIGURE 12. Plot of numerical velocity vectors calculated at $G r=5.0 \times 10^{4}, \operatorname{Pr}=3.0 \times 10^{-2}$ and $H a=100$ on $Y Z$-cross-sections at $x=1.0,2.0$ and 3.0.

are in agreement with the calculations of Ben Hadid \& Henry (1994) in a rectangular cavity in the case of $P r=0$, where predictions are limited to the velocity field.

In order to illustrate this point further, we show in figure 12 a plot of the velocity vectors taken from a simulation at $H a=100$. They are plotted for three transverse sections of the cavity at $x=1.0,2.0$ and 3.0. At this value of the Hartmann number, the boundary layers on the sidewalls of the cavity are thin and the flow is reduced to a regular two-dimensional circulation.

In the light of the above numerical results, the effect of the magnetic field on the structure of the flow was investigated experimentally. Temperature distributions were measured on a $Y Z$-cross-section in the cavity located at $x=3.0$, first for $H a=0$ then for $H a=64$, using the procedure described in $\S 3$. The Grashof number was set to a relatively high value, $G r=1.3 \times 10^{5}$, and the Prandtl number was set to $\operatorname{Pr}=2.3 \times 10^{-2}$, so that the measured isotherms would be significantly curved, as discussed in \$4.2.1. Temperature surfaces, calculated from the resulting data, are presented in figure 13(a) for $H a=0$ and figure $13(b)$ for $H a=64$. For each set of 

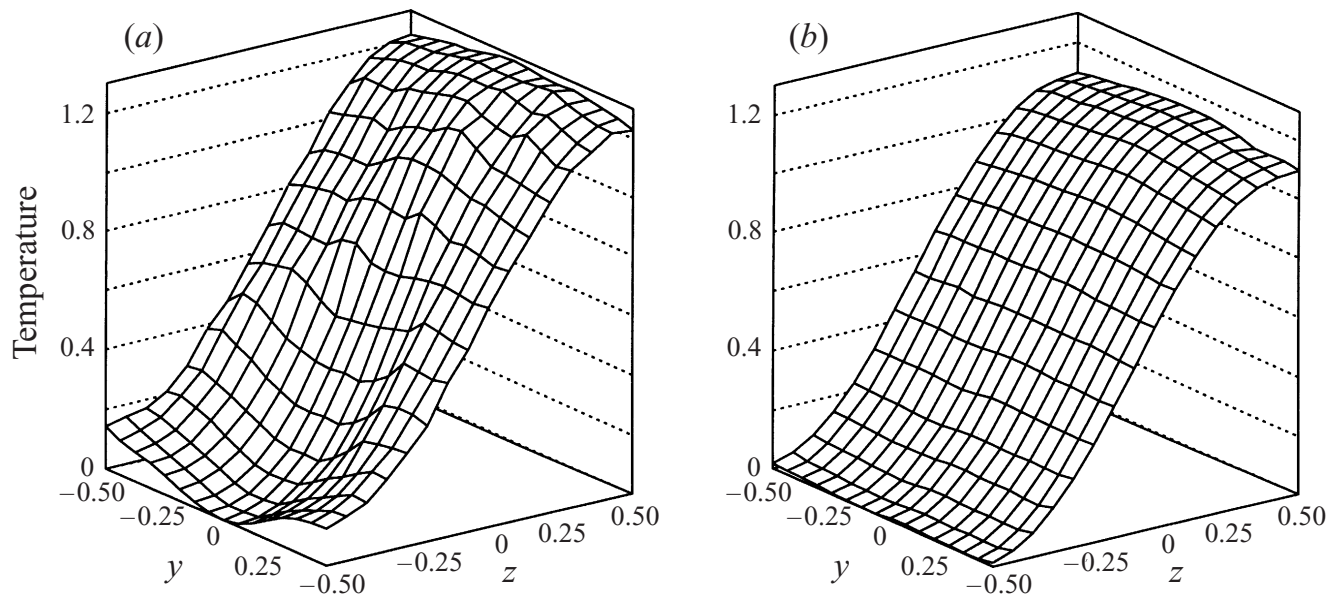

FigURE 13. Experimental temperature distributions on the $Y Z$-plane at $x=3.0(a) H a=0$,

(b) $\mathrm{Ha}=64$. The measurements were made for $\mathrm{Gr}=1.3 \times 10^{5}$ and $\mathrm{Pr}=2.3 \times 10^{-2}$.

measurements, the zero temperature point was set at the bottom measurement station situated at $y=0$.

In figure $13(a)$, the irregularity of the contours indicates that the flow is unsteady as discussed in $\S 4.2 .1$. The vertical temperature difference was strongest at $y=0$ and although the flow was time-dependent, the distribution is left-right symmetric. When a magnetic field is applied corresponding to $\mathrm{Ha}=64$, it alters the temperature distribution shown in figure $13(b)$. It can be seen that the three-dimensional character of the temperature is strikingly reduced so that the transverse structure of the temperature distribution is flattened and the variation of the temperature essentially restricted to the $z$-direction. Also, a substantial reduction of the vertical temperature stratification reflects the damping of the convective flow as discussed in $\$ 4.1$. Similar measurements to those presented in figure 13(a) were also taken at the lower Grashof number of $G r=9.8 \times 10^{4}$. They showed a weaker vertical temperature stratification than those displayed in figure $13(b)$ in the presence of a magnetic field. However, the temperature distribution was strongly three-dimensional suggesting that the modification to the flow field by the applied magnetic field is more than a simple reduction of convection, which could equally be obtained by reducing $G r$. It should also be noted that the magnetic field damps the temperature oscillations, as previously mentioned in $§ 4.2 .1$. This raises the question of whether the damping of the oscillatory flow is due to the modification of the averaged base flow or to a direct interaction between the magnetic field and the three-dimensional unsteady flow. This is a topic of current research.

\subsection{Comparison between the magnetohydrodynamic flow and the two-dimensional free convective circulation}

We showed in $\$ 4.2 .2$ that the three-dimensional convective circulation is restricted to two-dimensional motion in the $x$ - and $z$-directions at high Hartmann numbers. It is interesting to compare and contrast the magnetohydrodynamic flow with the two-dimensional model of free convection.

We show velocity vector plots obtained from the numerical simulations of the two different configurations in figure 14. Those obtained from a three-dimensional simulation of magnetohydrodynamic flow at $H a=100$ are shown plotted in the central $X Z$-plane in figure 14(a) while those from the two-dimensional free convection 
(a)

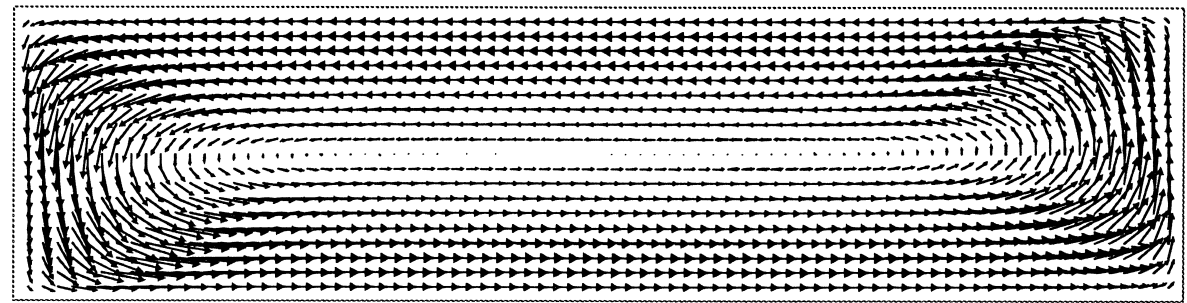

(b)

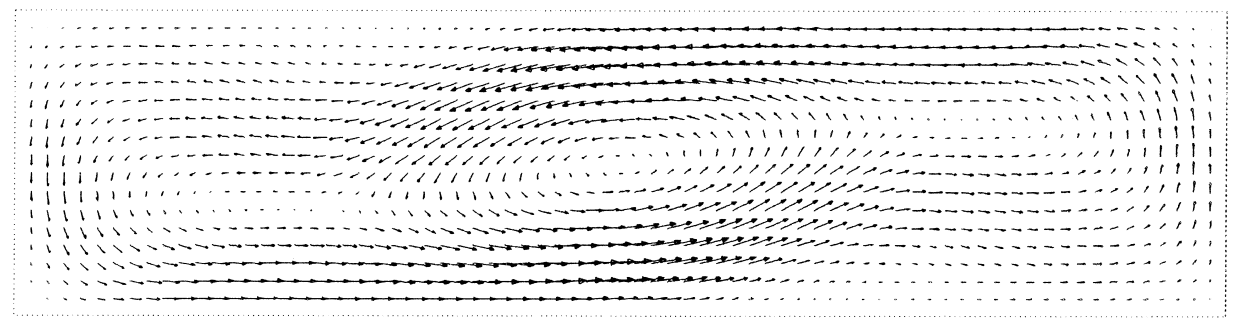

FIGURE 14. Comparison between the magnetohydrodynamic flow and the two-dimensional free convection model at $\mathrm{Gr}=5.0 \times 10^{4}$ and $\operatorname{Pr}=3.0 \times 10^{-2}:(a)$ three-dimensional numerical simulations at $H a=100,(b)$ two-dimensional numerical simulations.

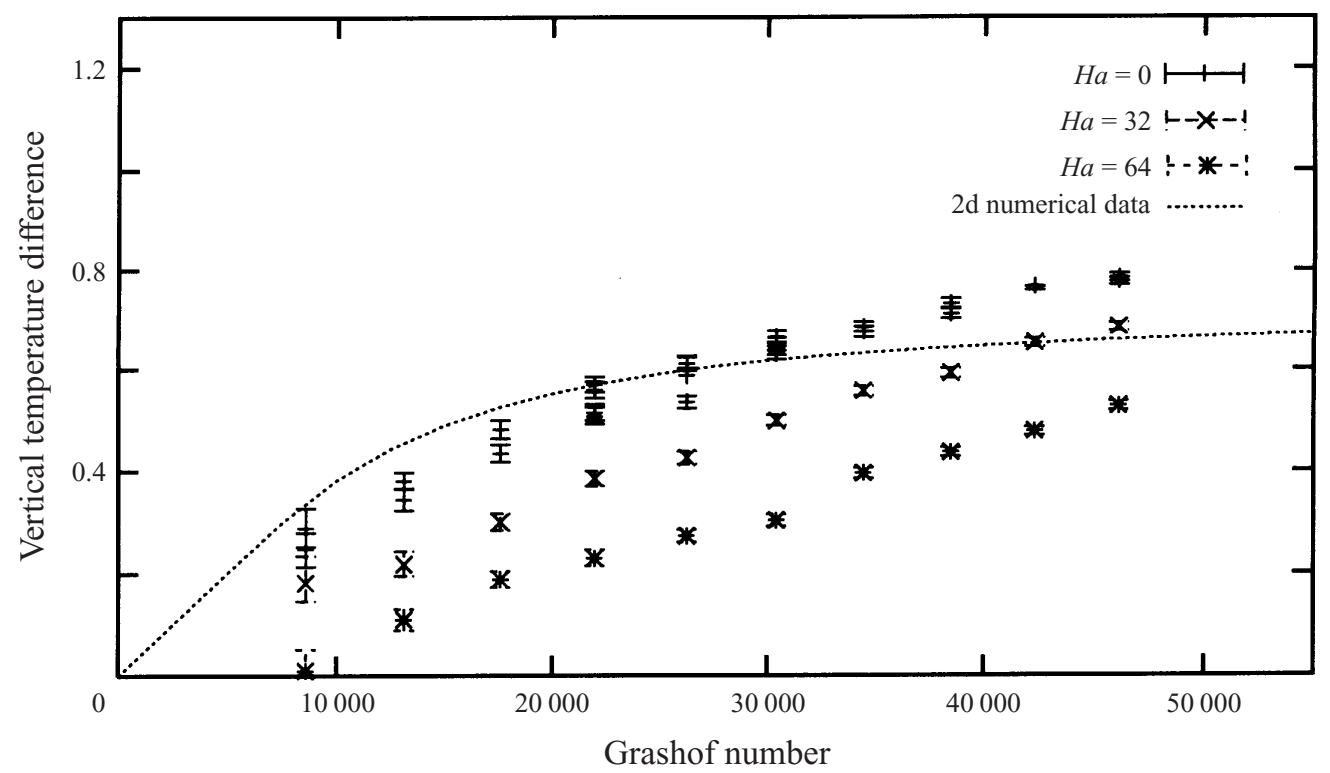

FIGURE 15. Vertical temperature difference versus the Grashof number: comparison between experimental measurements at $H a=0, H a=32$ and $H a=64$ and two-dimensional simulations performed at $\operatorname{Pr}=3.0 \times 10^{-2}$

solution are presented in figure 14(b). In the two-dimensional case, the flow consists of a large tilted convection cell which is centro-symmetric. Thus, it can be seen that the two flows obtained at the same fixed $\mathrm{Gr}$ and $\mathrm{Pr}$ are qualitatively different.

The remaining investigation focuses on whether the heat transfer characteristics of the magnetohydrodynamic flow are similar to those of the free convection model 
over a range of Grashof numbers. We carried this out by measuring the experimental dependence of the vertical temperature difference $\Theta$ in the centre of the cavity $(x=2.0, y=0)$ for $H a=0, H a=32$ and $H a=64$ and comparing them with two-dimensional numerical results previously reported in Braunsfurth et al. (1997). The results are displayed in figure 15. It is immediately clear that the experimental flow in the presence of a uniform transverse magnetic field remains qualitatively different from the two-dimensional model since all three experimental curves show systematic differences with the numerical one. Finally, it is interesting to note that at these relatively small temperature differences, an applied magnetic field has a strong effect on the temperature distribution. However, this does not necessarily indicate a similar effect on the flow, as the Prandtl number of gallium is small.

\section{Conclusion}

It has been known for more than thirty years that magnetic fields can be used to suppress oscillations in liquid metal flows. Despite this, there is little use of this technique in the crystal growth industry. Perhaps one reason that this approach has not advanced is the lack of detailed knowledge of the fundamental fluid mechanics of the MHD processes involved. We have shown using the powerful combination of experimental and numerical techniques that the base flow state in our model of a crystal growth facility is modified into a new two-dimensional configuration. Oscillations are thereby suppressed and it remains an interesting challenge to theory to test the stability of this new state. We believe this will provide important insight into mechanisms for selectively controlling flows using modest magnetic fields instead of attempting to suppress convection completely. An example of this has already been provided by McKell et al. (1990) who showed that low-dimensional chaos could be promoted using a small field. The converse of this may also be true.

The work of A.J. was supported by a studentship funded by DERA Malvern and the collaborative research is supported by the British Council under the Alliance Program.

\section{REFERENCES}

Afrid, M. \& ZEBib, A. 1990 Oscillatory three-dimensional convection in rectangular cavities and enclosures. Phys. Fluids A 2, 1318-1327.

Alboussière, T., Garandet, J. P. \& Moreau, R. 1993 Buoyancy-driven convection with a uniform magnetic field. Part I. Asymptotic analysis. J. Fluid Mech. 253, 545-563.

Alboussière, T., Garandet, J. P. \& Moreau, R. 1996 Asymptotic analysis and symmetry in MHD convection. Phys. Fluids 8, 2215-2226.

AlchaAR, S., Vasseur, P. \& Bilgen, E. 1995 Natural convection heat transfer in a rectangular enclosure with a transverse magnetic field. Trans. ASME: J. Heat Transfer 117, 668-673.

Ben Hadid, H. \& Henry, D. 1994 Numerical simulation of convective three-dimensional flows in a horizontal Bridgman configuration under the action of a constant magnetic field. Proc. Second Intl Conf. on Energy Transfer in Magnetohydrodynamic Flows, Aussois, France, vol. 1, pp. 47-56. Pamir Publications.

BEN HADID, H. \& Henry, D. 1996 Numerical simulation of convective three-dimensional flows in a horizontal cylinder under the action of a constant magnetic field. J. Crystal Growth 166, 436-445.

Ben Hadid, H. \& Henry, D. 1997 Numerical study of convection in the horizontal Bridgman configuration under the action of a constant magnetic field. Part 2. Three-dimensional flow. J. Fluid Mech. 333, 57-83. 
Braunsfurth, M. G. \& Mullin, T. 1996 An experimental study of oscillatory convection in liquid gallium. J. Fluid Mech. 327, 199-219.

Braunsfurth, M. G., Skeldon, A. C., Juel, A., Mullin, T. \& Riley, D. S. 1997 Free convection in liquid gallium. J. Fluid Mech. 342, 295-314.

Daniels, P. G., Blythe, P. A. \& Simpkins, P. G. 1987 Onset of multicellular convection in a shallow laterally heated cavity. Proc. R. Soc. Lond. A 114, 327-350.

Davoust, L. 1996 Convection naturelle MHD dans une cavité horizontale élancée. PhD Thesis, Institut National Polytechnique de Grenoble, France.

Davoust, L., Moreau, R., Bolcato, R., Alboussière, T., Neubrand, A. C. \& Garandet, J. P. 1995 Influence of a vertical magnetic field on convection in the horizontal Bridgman crystal growth configuration. Magnetohydrodynamics 31, 218-227.

Garandet, J. P., Alboussière, T. \& Moreau, R. 1992 Buoyancy-driven convection in a rectangular enclosure with a transverse magnetic field. Intl J. Heat Mass Transfer 35, 741-748.

GiLl, A. E. 1966 The boundary-layer regime for convection in a rectangular cavity. J. Fluid Mech. 132, 271-281.

Hunt, J. C. R. \& Shercliff, J. A. 1971 Magnetohydrodynamics at high Hartmann number. Ann. Rev. Fluid Mech. 3, 37-62.

Hurle, D. T. J. 1966 Temperature oscillations in molten metals and their relationship to growth striae in melt-grown crystals. Phil. Mag. 13, 305-310.

Hurle, D. T. J., Jakeman, E. \& Johnson, C. P. 1974 Convective temperature oscillations in molten gallium. J. Fluid Mech. 64, 565-576.

Juel, A., Mullin, T., Ben Hadid, H. \& Henry, D. 1998 In preparation for submission to J. Fluid Mech.

LANGLOIS, W. E. 1985 Buoyancy-driven flows in crystal-growth melts. Ann. Rev. Fluid Mech. 17, 191-215.

McKell, K. E., Broomhead, D. S., Jones, R. \& Hurle, D. T. J. 1990 Torus doubling in convecting molten gallium. Europhys. Lett. 12, 513-518.

Moreau, R. 1990 Magnetohydrodynamics. Kluwer.

Müller, G. \& Ostrogorsky, A. 1993 Convection in melt growth. In Handbook of Crystal Growth, vol. 2b, Bulk Crystal Growth: Growth Mechanisms and Dynamics (ed. D. T. J. Hurle). NorthHolland.

Neubrand, A. C., Garandet, J. P., Moreau, R. \& Alboussière, T. 1995 Influence of a slight non-uniformity of the magnetic field on MHD convection. Magnetohydrodynamics 31, 1-17.

OKADA, K. \& OzOE, H. 1992 Experimental heat transfer rates of natural convection of molten gallium suppressed under an external magnetic field in either the $\mathrm{X}, \mathrm{Y}$, or $\mathrm{Z}$ direction. J. Heat Transfer 114, 107-114.

Oreper, G. M. \& SzeKely, J. 1983 The effect of an externally imposed magnetic field on buoyancydriven flow in a rectangular cavity. J. Crystal Growth 64, 505-515.

Ozoe, H. \& OKadA, K. 1989 The effect of the direction of the external magnetic field on the three-dimensional natural convection in a cubical enclosure. Intl J. Heat Mass Transfer 32 1939-1954.

Roux, B. (Ed.) 1990 GAMM Workshop 1990: Numerical Simulation of Oscillatory Convection in Low Pr Fluids. Notes on Numerical Fluid Mechanics vol. 27, Vieweg.

Series, R. W. \& Hurle, D. T. J. 1991 The use of magnetic fields in semiconductor crystal growth. J. Crystal Growth 113, 305-328.

SingH, K. R. \& Cowling, T. G. 1963 Thermal convection in magnetohydrodynamics. II Flow in a rectangular box. Q. J. Mech. Appl. Maths 16, 17-31.

Venkatachalappa, M. \& Subbaraya, C. K. 1993 Natural convection in a rectangular enclosure in the presence of a magnetic field with uniform heat flux from the sidewalls. Acta Mechanica 96, $13-26$. 